\title{
A New Generating Function for Aunu Patterns: Applications in Integer Group Modulo $n$
}

\author{
${ }^{* 1}$ A. Usman and ${ }^{2}$ A.A. Ibrahim \\ ${ }^{1}$ Department of Mathematics and Computer Sciences, \\ Umaru Musa Yar'adua University, P.M.B. 2218 Katsina, Nigeria. \\ Department of Mathematics, Usmanu Danfodiyo University, P.M.B. 2346 Sokoto, Nigeria \\ [*Corresponding Author: E-mail-_maminukbai@yahoo.com]
}

ABSTRACT: This paper identified a new and more generalised generating function for the Aunu Patterns which is based on the methods employed earlier by Garba. It also identified and discussed some other theoretic properties of the Aunu Patterns and Aunu Groups especially in relation to integer modulo groups.

Keywords: Aunu Groups, Aunu Patterns, Aunu Numbers, Cycles, Permutations, Symmetric Groups.

\section{INTRODUCTION}

The concept of a group arose from the study of polynomial equations, starting with Evariste Galois in 1830s. Modern group theory is a very active mathematical discipline and mathematiccians have devised various methods of both theoretical and computational point of view.

The modern concept of abstract group was developed out of several fields of mathematics (Smith, 1906; Kleiner, 1986; Wussing, 2007). The original motivation for group theory was the search for solutions of polynomial equations of degree greater than 4. The 19th-century French mathematician Évariste Galois, extending prior work of Paolo Ruffini and Joseph-Louis Lagrange, gave a criterion for the solvability of a particular polynomial equation in terms of the symmetry group of its roots (solutions). The elements of such a Galois group correspond to certain permutations of the roots. At first, Galois' ideas were rejected by his contemporaries, and published only after his death, Arthur (1889) and Galois (1908).
Permutation groups were investigated in particular by Augustin Louis Cauchy. Cayley (1889) also investigated permutation groups, as depending on the symbolic equation $\theta^{n}=1$. According to Ibrahim (2007), Cayley gave the first abstract definition of a group,.

Ibrahim, (2004) denote $I_{5}$ as the group generated from Aunu patterns. This is an alternative way of constructing the Bara'at-alDhimmah model using permutation mapping $\alpha$ on the set $\dot{Y}_{5}$. Abor and Ibrahim (2010) developed a scheme for the range of such cycles and used it to investigate further number theoretic and algebraic properties of $G_{P}$ and $X$ as applications. This scheme was motivated by the nature of the sequence generated in the pairing of points of the cycles. They used the concept of Catalan numbers to develop the scheme for prime numbers $P \geq 5$ and $\Omega \subseteq \mathbb{N}$ which generate the cycles of Aunu patterns designed as follows.

$$
\omega_{i}=\left((1)^{m p}(1+i)^{m p}(1+2 i)^{m p}(1+3 i)^{m p}(1+4 i)^{m p} \ldots(1+(p-1))^{m p}\right)
$$

The organization in the paper consists first, of the basic concepts and definitions concerning the key notions discussed. This is followed by the method of construction of the desired generating function and subsequently, the evolution of group structure as application.

Notation: We shall denote as follows:

Example 1. Permutation that map $1 \ddot{Y}$ 2, $2 \ddot{Y} 3$, $3 \ddot{Y} 4,4 \ddot{Y} 5$ and $5 \ddot{Y} 1$, can be represented as $\langle 12345\rangle$. $\bmod p:$ is the usual integer modulo. In this context, it is used as a restriction parameter for the natural numbers as contained in the class of permutation.

\langle\rangle : represents the cycles of a class of permutation.

$p:$ means a prime number greater than or equal to 5 . That is, $p=5,7,11,13, \ldots$ 
Denote $A_{p}$ : The group generation from Aunu group.

$A_{n(p)}$ : Element (cycle) displaying a particular pattern of $n$ elements. For brevity we shall denote such a pattern as Aunu pattern.
Construction of Generating Function for Aunu Patterns

We shall be guided by the following notation of symbols for obtaining the general function that determine the order of the patterns in the cyclic representation of Aunu group as a class of permutation.

Computation for $p=5$

$$
\begin{aligned}
& \langle 1,1+1, \ldots, 1+(p-1)\rangle=\left\langle 1^{\bmod p},(1+1)^{\bmod p}, \ldots,[1+(p-1)]^{\bmod p}\right\rangle \\
& \langle 1,1+2, \ldots, p-1\rangle=\left\langle 1^{\bmod p},(1+2)^{\bmod p}, \ldots,(p-1)^{\bmod p}\right\rangle \\
& \langle 1,1+3, \ldots, p-2\rangle=\left\langle 1^{\bmod p},(1+3)^{\bmod p}, \ldots,(p-2)^{\bmod p}\right\rangle \\
& \langle 1,1+4, \ldots, p-3\rangle=\left\langle 1^{\bmod p},(1+4)^{\bmod p}, \ldots,(p-3)^{\bmod p}\right\rangle
\end{aligned}
$$

\section{Computation for $p=7$}

$$
\begin{aligned}
& \langle 1,1+1, \ldots, 1+(p-1)\rangle=\left\langle 1^{\bmod p},(1+1)^{\bmod p}, \ldots,[1+(p-1)]^{\bmod p}\right\rangle \\
& \langle 1,1+2, \ldots,(p-1)\rangle=\left\langle 1^{\bmod p},(1+2)^{\bmod p}, \ldots,(p-1)^{\bmod p}\right\rangle \\
& \langle 1,1+3, \ldots,(p-2)\rangle=\left\langle 1^{\bmod p},(1+3)^{\bmod p}, \ldots,(p-2)^{\bmod p}\right\rangle \\
& \langle 1,1+4, \ldots,(p-3)\rangle=\left\langle 1^{\bmod p},(1+4)^{\bmod p}, \ldots,(p-3)^{\bmod p}\right\rangle \\
& \langle 1,1+5, \ldots,(p-4)\rangle=\left\langle 1^{\bmod p},(1+5)^{\bmod p}, \ldots,(p-4)^{\bmod p}\right\rangle \\
& \langle 1,1+6, \ldots,(p-5)\rangle=\left\langle 1^{\bmod p},(1+6)^{\bmod p}, \ldots,(p-5)^{\bmod p}\right\rangle
\end{aligned}
$$

\section{Computation for $\boldsymbol{n}$, a prime $\geq 7$}

$$
\begin{aligned}
& \langle 1,1+1, \ldots, 1+(p-1)\rangle=\left\langle 1^{\bmod p},(1+1)^{\bmod p}, \ldots,[1+(p-1)]^{\bmod p}\right\rangle \\
& \langle 1,1+2, \ldots,(p-1)\rangle=\left\langle 1^{\bmod p},(1+2)^{\bmod p}, \ldots,(p-1)^{\bmod p}\right\rangle \\
& \vdots \quad \vdots \quad \vdots \quad=\quad \vdots \quad \vdots \\
& \langle 1,1+(p-1), \ldots, p-(p+2)\rangle=\left\langle 1^{\bmod p},[1+(p-1)]^{\bmod p}, \ldots,[p-(p+2)]^{\bmod p}\right\rangle
\end{aligned}
$$

Combining the above general notation with Abor, (2009)

(i.e. $\left.\omega_{i}=\left(1^{\bmod p}(1+i)^{\bmod p}(1+2 i)^{\bmod p}(1+3 i)^{\bmod p} \ldots(1+(p-1))^{\bmod p}\right)\right) \quad$ we obtain the general notation which generate all the cycles of prime cardinality in Aunu numbers

$$
A_{n}=\left\langle 1^{\bmod p},(1+n)^{\bmod p},(1+2 n)^{\bmod p},(1+3 n)^{\bmod p}, \ldots,[1+(p-n)]^{\bmod p}\right\rangle \text {, for } n=1,2, \ldots,(p-1) \text {. }
$$

\section{Construction of Some Group Structures Using Aunu Patterns.}

Definition (Permutations): a bijection $f: S \rightarrow S$ is called a permutation of $S$, where $S=\{1,2,3, \ldots, \mathrm{n}\}$. Consider the set $S$ of the $n$ ! permutations of these $n$ symbols, a permutation of a set $S$ is a one-to-one function from $S$ onto $S$ (No significance is to be given to the fact that they are natural numbers). First, however, we shall introduce useful examples of permutation.

Example 2: Let (12345) (13524) (14253) (15432) be some cyclic arrangements of the elements of S. We now introduce for these permutations a table of composition in Table 1 . 
Table 1: Composition of Function for the Aunu Patterns

\begin{tabular}{lllll}
\hline$\circ$ & $(12345)$ & $(13524)$ & $(14253)$ & $(15432)$ \\
\hline$(12345)$ & $(13524)$ & $(14253)$ & $(15432)$ & $(1)$ \\
$(13524)$ & $(14253)$ & $(15432)$ & $(1)$ & $(12345)$ \\
$(14253)$ & $(15432)$ & $(1)$ & $(12345)$ & $(13524)$ \\
$(15432)$ & $(1)$ & $(12345)$ & $(13524)$ & $(14253)$ \\
\hline
\end{tabular}

From Table 1 the following compositions are achievable:

$$
\begin{aligned}
& (12345)(13524)=(13524)(12345)=(14253) \\
& (12345)(14253)=(14253)(12345)=(15432) \\
& (12345)(15432)=(15432)(12345)=(1) \\
& (13524)(14253)=(14253)(13524)=(1) \\
& (13524)(15432)=(13524)(15432)=(12345) \\
& (14253)(15432)=(15432)(14253)=(13524) .
\end{aligned}
$$

It follows that the binary operation in Table 1 is commutative and since these cycles generate a group structure, Ibrahim, (2004) it is clear, the group is also an abelian group.

Note: If $\alpha, \beta \in S_{n}$ are disjoint cycles, then $\alpha \beta=\beta \alpha$.

The cycles in table 1 of composition are disjoint cycles, Since;

$(12345)(13524)=(13524)(12345)=(14253)$

$(14253)(15432)=(15432)(14253)=(13524)$.

If an element $\{(1)\}$ is embedded in the table 1, i.e.

$(\{(12345),(13524),(14253),(15432)\} \cup\{(1)\})$ we obtain a group. In that case $e=(1)$ represents the identity element.

In the original construction Ibrahim (2004), this group was denoted $I_{5}$, while in Abor, (2009) it was denoted $\omega_{i}$. For brevity, we shall denote our construction $A_{p}, p \geq 5$. That is,

$$
A_{5}=\{(12345),(13524),(14253),(15432)\} .
$$

It is evident, the existence of inverse elements for all $x \in A_{5}$. Thus:

(12345) is the inverse element of (15432), and (13524) is the inverse element of (14253).

If $x, y, z \in A_{P}$ for $p \geq 5$. Then $(x \circ y) \circ z=x \circ(y \circ z)$. From Table 1 the results are as follows: $((12345)(13524))(14253)=(12345)((13524)(14253))$. That is:

For L.H.S. we have $((12345)(13524))(14253)=(14253)(14253)=(14253)^{2}=(12345)$. and for R.H.S. we obtain $(12345)((13524)(14253))=(12345)(1)=(12345)$.

Hence, L.H.S = R.H.S. associativity hold.

So, the above results obtained from the permutations (Table 1) of composition, have satisfied all the properties of a group. Therefore, we conclude that the table generates a group.

The generating set of Table 1 is as follows: 


$$
\begin{aligned}
& S=\langle(12345), \quad(13524), \quad(14253), \quad(15432)\rangle . \text { That is: } \\
& (12345)^{1}=(12345) \\
& (12345)^{2}=(12345)(12345)=(13524) \mathrm{s} \\
& (12345)^{3}=(12345)^{2}(12345)=(13524)(12345)=(14253) \\
& (12345)^{4}=(12345)^{3}(12345)=(14253)(12345)=(15432) \\
& (12345)^{5}=(12345)^{4}(12345)=(15432)(12345)=(1) .
\end{aligned}
$$

The same results can be obtained using (13524), (14253), (15432).

This indicated that the permutations (Table 1) above form a cyclic group.

Note: a generating set can never contain the identity element. This is because the identity can only generate itself.

Construction of Additive Group: Using the Table 1 , we can form an additive group table. The Tables 2-3 illustrates the construction process of a group structure using additive notation and denoting cycles as positive integers.

Table 2: Additive notation Using Table 1

\begin{tabular}{lllll}
\hline+ & $\mathbf{1}$ & $\mathbf{2}$ & $\mathbf{3}$ & $\mathbf{4}$ \\
\hline $\mathbf{1}$ & 2 & 3 & 4 & 0 \\
$\mathbf{2}$ & 3 & 4 & 0 & 1 \\
$\mathbf{3}$ & 4 & 0 & 1 & 2 \\
$\mathbf{4}$ & 0 & 1 & 2 & 3 \\
\hline
\end{tabular}

Table 3: Embedding of Identity element to Table 2

\begin{tabular}{llllll}
\hline+ & $\mathbf{1}$ & $\mathbf{2}$ & $\mathbf{3}$ & $\mathbf{4}$ & $\mathbf{0}$ \\
\hline $\mathbf{1}$ & 2 & 3 & 4 & 0 & 1 \\
$\mathbf{2}$ & 3 & 4 & 0 & 1 & 2 \\
$\mathbf{3}$ & 4 & 0 & 1 & 2 & 3 \\
$\mathbf{4}$ & 0 & 1 & 2 & 3 & 4 \\
$\mathbf{0}$ & 1 & 2 & 3 & 4 & 0 \\
\hline
\end{tabular}

The Table 2 forms a symmetric group of order $p-1$ and is isomorphic to Table 1. If zero elements $\{0\}$ are embedded in the above Table i.e. $(\{1,2,3,4\} \cup\{0\})$ we obtain a symmetric group of order $p$ as given in the Table 3. Thus, Table 3 form an additive group of order $p$

\section{CONCLUSION}

This paper has provided a useful conversion process from the cyclic elements of the group generated using Aunu groups using additive group of integers modulo $\mathrm{n}$ where $\mathrm{n}$ is necessarily a prime. It has also provided a method of construction of the generating function that can be used in the formulation of the Aunu patterns and subsequently, the evolution of group structure as application. It also highlighted the application of theoretic properties of Aunu group.

\section{REFERENCES}

Abor, G.I. (2009). A New Method of Constructing a Variety of Finite Group Based on Some Succession Scheme, PhD Seminar.

Abor G.I. and Ibrahim A.A. (2010). Some Algebraic and Topological Properties of Groups with Prime Order (Unpublished).

Cayley, A. (1889). The Collected Mathematical Papers of Arthur Carley, II (1851-1860), Cambridge University Press.

Galois, E (1908). Tarinery, Jules, ed. Manuscrits de Evariste Galois [Evariste Galoisô Manuscripts, Paris: Gauthier-Villars.]

Kleiner, I. (1986). The Evolution of Group Theory: A Brief Survey, Math. Mag. 59(4): 195-215.

Ibrahim, A.A. (2004). Group Theoretical Interpretation of Bara'at al-Dhimmah Models for Prayers that are not Strictly Consecutive. Proceedings of Annual National Conference of the Mathematical Association of Nigeria, 35--46.

Ibrahim, A.A. (2007). A Counting Scheme and Some Algebraic Properties of a Class of Special Permutation Patterns. J. Discrete Math. Sci. Cryptograph 10(4): 537-546.

Smith, D.E. (1906). History of Modern Mathematics, Mathematical Monographs, No.1.

Wussing H. (2007), The Genesis of the Abstract Group Concept: A Contribution to the History of the origine of Abstract group Theory, New York: Dover Publications, ISBN 978-0-486-45868-7. 